\title{
Increased expression of vascular endothelial growth factor associated with accumulation of lipids in Bruch's membrane of $L D L$ receptor knockout mice
}

\author{
M Rudolf, B Winkler, Z Aherrahou, L C Doehring, P Kaczmarek, U Schmidt-Erfurth
}

Aim: To investigate the pathogenesis of age related macular degeneration (ARM) with respect to lipid accumulation within Bruch's membrane $(B r M)$ in a knockout model with low density lipoprotein (LDL) receptor deficiency.

Methods: LDL receptor deficient mice and C57BL/6 controls were fed a standard diet or a high fat (HF) diet. Plasma total cholesterol (pTC) was determined. Eyes were examined by transmission electron microscopy. Immunohistochemical staining for VEGF was performed.

Results: pTC were highest in LDL receptor deficient mice after $\mathrm{HF}$ diet and elevated after standard diet compared to controls with and without HF diet. While BrM of controls did not exhibit any visible changes, membrane bound translucent particles were seen in all BrM of knockout mice. The amount of these particles was substantially increased and membranes were thickened after HF diet. VEGF staining was positive in knockout mice only and was located in retinal pigment epithelial cells, the outer plexiform layer, and photoreceptor inner segments. Most intensive VEGF expression was documented after HF diet.

Conclusion: LDL receptor deficient mice exhibit an accumulation of lipid particles in $\mathrm{BrM}$ which is further increased after fat intake. VEGF expression is found in the outer retinal layers of $L D L$ receptor deficient mice and appears to correlate with the amount of lipid particles present in $\mathrm{BrM}$.

A ge related maculopathy (ARM) is the most frequent cause of severe visual impairment in the elderly in industrial countries. In these patients, most dramatic irreversible vision loss occurs as a result of subretinal choroidal neovascularisation (CNV). ${ }^{1}$

The pathogenesis of ARM is poorly understood. Lesions that involve retinal pigment epithelium (RPE) and Bruch's membrane (BrM) are the most prominent clinical and histopathological features of ARM..$^{2-4}$ Recent findings highlight a role for lipids. In normal human retina, neutral lipids accumulate in BrM throughout life. ${ }^{5}$ In some epidemiological studies atherosclerosis has been associated with ARM, but a correlation was not consistently found in all surveys. ${ }^{16-8}$ Also risk factors for atherosclerosis, such as advanced age, hypercholesterolaemia, smoking, high intake of saturated fat, and decreased oestrogen exposure, have been associated with ARM in other studies. ${ }^{178}$

In this study we used a well established atherosclerotic murine model to evaluate changes in BrM and associated biological effects caused by elevated plasma total cholesterol (pTC) levels.

LDL (low density lipoprotein) receptor deficient mice (C57BL/6J background) are not able to incorporate plasma cholesterol into body cells sufficiently, resulting in increased pTC even under standard diet. Our controls were C57BL/6J mice without any receptor deficiency. pTC levels were furthermore modified by different diets varying in fat content. Using this setting we already observed distinctive lipid rich degenerations of BrM. ${ }^{9}$

Vascular endothelial growth factor (VEGF) was identified as one of the major stimuli in experimental CNV. ${ }^{10}$ We performed immunohistochemical staining for VEGF in normal and knockout animals to identify its expression and distribution patterns and its eventual correlation with BrM changes.

\section{MATERIALS AND METHODS}

The use of animals was in accordance with the ARVO statement for the use of animals in ophthalmic and vision research. Ethics committee institutional review board approval was obtained.

Female wild type C57BL/6J and LDL receptor deficient C57BL/6J-LDL-r(-/-) mice were perchased (Jackson Laboratories, Bar Harbor, ME, USA).

Animals were maintained in plastic cages with even lightdark cycle with permanently free access to water and food. At the age of 2 months, regular chow ( $12 \%$ of calories as fat) was changed in one half of C57BL/6J $(\mathrm{n}=6)$ and $\mathrm{C} 57 \mathrm{BL} / 6 \mathrm{~J}$ LDL-r $(-/-) \quad(\mathrm{n}=6)$ population to high fat $(\mathrm{HF})$, high cholesterol diet western style (TD 90221, Harlan-Teklad, Madison, WI, USA) containing $30 \%$ of calories as fat. All other C57BL/6J $(\mathrm{n}=6)$ and C57BL/6J-LDL-r $(-/-) \quad(\mathrm{n}=6)$ continued their regular chow diet. Both diets contained comparable mineral and vitamin mixes.

\section{Plasma cholesterol levels}

Heparins blood was obtained by retro-orbital bleeding from anaesthetised mice before sacrifice. pTC and triglyceride concentrations were measured using standard colorimetric assays purchased from Sigma (Deisenhofen, Germany) and Roche Diagnostics (Hamburg, Germany).

\section{Tissue preparation}

At the age of 4 months all animals were anaesthetised with isoflurane and sacrificed by cervical dislocation. Eyes were enucleated immediately. Whole eyes were fixed in 2.5\% glutaraldehyde in $0.1 \mathrm{M}$ cacodylate- $0.2 \mathrm{M}$ sodium phosphate buffer, postfixed in $1 \%$ osmium tetroxide, stained with $1 \%$ uranyl acetate, and embedded in epoxy resin (Epon 812).

\footnotetext{
Abbreviations: ARM, age related macular degeneration; BrM, Bruch's membrane; CNV, choroidal neovascularisation; HF, high fat; IPS, inner photoreceptor segments; LDL, low density lipoprotein; OPL, outer plexiform layer; $\mathrm{pTC}$, plasma total cholesterol; RPE, retinal pigment epithelium; TEM, transmission electron microscopy; VEGF, vascular endothelial growth factor
} 
Table 1 Plasma lipid levels in C57BL/6 and LDL receptor deficient mice

\begin{tabular}{|c|c|c|c|c|}
\hline \multirow[b]{2}{*}{ Mouse } & \multirow[b]{2}{*}{ Diet } & \multicolumn{2}{|l|}{ Plasma total } & \multirow{2}{*}{$\frac{\text { LDL+VLDL }}{\text { Cholesterol }}$} \\
\hline & & Cholesterol & Triglycerides & \\
\hline C57BL/6J & chow & $82.0(19.5)$ & $29.2(6.0)$ & 28.0 (15.9) \\
\hline C57BL/6J & high fat & $119.2(36.5)$ & $36.0(3.1)$ & $109.0(44.3)$ \\
\hline C57BL/6 ldlr-/- & chow & 189.0 (37) & $72.7(20.6)$ & $125.7(29.3)$ \\
\hline C57BL/6 ldlr-/- & high fat & 498.3 (134.3) & $137.8(44.8)$ & 443.8 (124.7) \\
\hline
\end{tabular}

Data are expressed in mean (SD) mg/dl, LDL, low density lipoprotein; VLDL, very low density lipoprotein; $n=6$ in each group.

Eyes were sectioned in $1 \mu \mathrm{m}$ on an ultramicrotome. The tissue sections were stained with $2 \%$ toluidine blue $\mathrm{O}$ and examined under light microscope.

\section{Transmission electron microscopy}

After determining areas of interest, thin sections of approximately 50-90 nm were cut, collected on copper grids and stained with $4 \%$ uranyl acetate and lead citrate. Subsequently, the sections were evaluated by transmission electron microscopy (TEM, Carl Zeiss EM9, Oberkochen, Germany) and photographed.

\section{Immunohistochemical analysis}

Tissue sections were stained using the streptavidin-biotin method with staining agent AEG (red) and Mayer's haemalaun for nucleus staining (blue). The purified Gl43850 antibody (Mouse $\operatorname{IgG}_{2 a}$, PharMingen, San Diego, USA) has been used to immunoprecipitate native VEGF ( $1 \mathrm{~g} \mathrm{mAb} /$ $50 \mathrm{ml}$ lysate) and to identify three VEGF isoforms (165, 189, 206aa).

Statistical analysis

Mean plasma cholesterol levels were calculated. The Wilcoxon rank sum test was performed to determine statistically significant differences in cholesterol levels according to diet and LDL receptor deficiency.

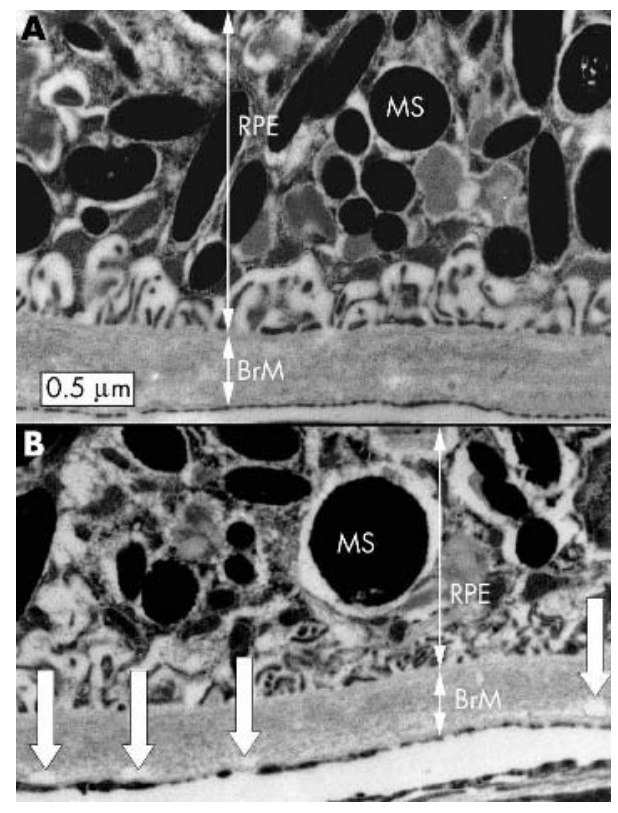

Figure 1 TEM: (A) 4 months old control mice (C57Bl/6J) after 2 months of high fat diet. Homogeneous Bruch's membrane (BrM) with regular structure, retinal pigment epithelium (RPE), melanosomes (MS). (B) 4 months old LDL receptor deficient mice (C57BI/6J-LDL-r(-/-)) after chow diet, Bruch's membrane with regular architecture but with basal membrane bound translucent vesicles (arrows); scale: white bar $=0.5 \mu \mathrm{m}$.

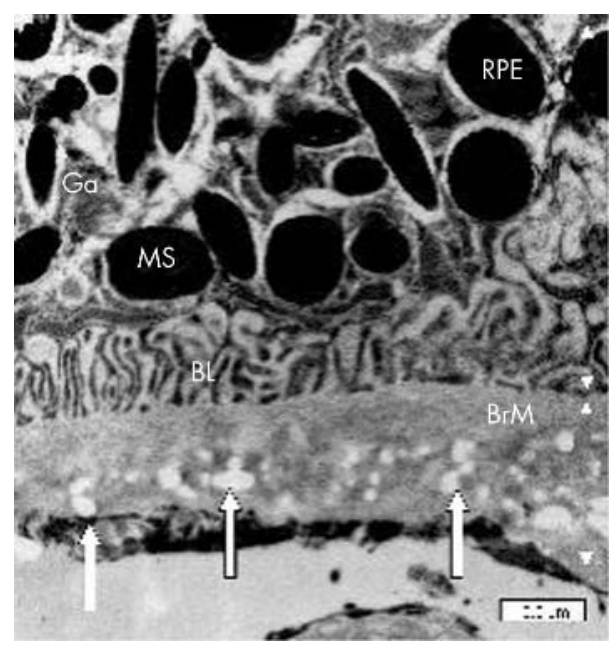

Figure 2 TEM: 4 months old LDL receptor deficient mice (C57B)/6JLDL-r(-/-)) after 2 months of high fat diet. Bruch's membrane (BrM) is substantially thickened with beginning condensation of collagenous and elastic fibres. The number of membrane bound translucent vesicles is increased with additional deposits of non-membrane bound particles. The non-membrane bound electron lucent particles were round and occasionally confluent. (arrows) Retinal pigment epithelium (RPE), melanosomes (MS); scale: white bar $=0.5 \mu \mathrm{m}$.

\section{RESULTS}

pTC levels

pTC levels were highest in LDL receptor deficient mice after HF diet $(p=0.0121)$ and significantly elevated after chow diet $(p=0.032)$ compared to control mice with $(p=0.024)$ and without HF diet (table 1).

\section{Bruch's membrane changes in TEM}

Eleven of 12 control animals did not exhibit any visible changes in BrM by TEM even after HF diet (fig lA). In contrast, membrane bound translucent particles were seen in membranes of all 12 LDL receptor deficient mice $(+1$ control after HF) (figlB).

Additionally, in all six knockout mice following HF diet membranes were substantially thickened with a beginning condensation of collagenous and elastic fibres. In this group the number of translucent vesicles was also increased with additional deposits of non-membrane bound particles (fig 2) which were round, occasionally confluent, and scattered throughout both collagenous layers.

\section{VEGF immunohistology}

No significant VEGF expression was detected in all 12 control mice irrespectively of diet (+l knockout after chow) (fig 3A). However, positive staining for VEGF was found in 11 of 12 LDL receptor deficient mice and was predominantly located in basal RPE, the outer plexiform layer and photoreceptor inner segments (fig 3B). Highest intensity in 


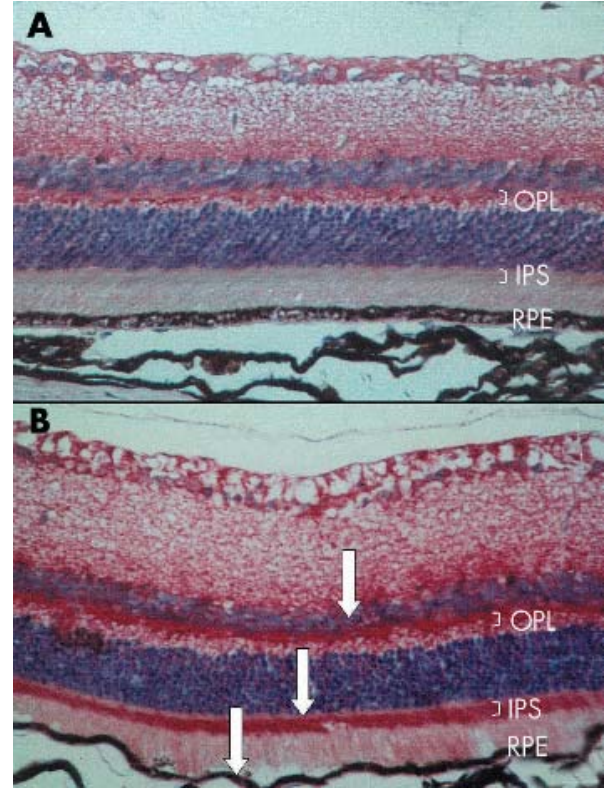

Figure 3 VEGF immunohistology: (A) 4 months old control mice (C57Bl/6J) after chow diet, no changes were observed after VEGF antibody incubation. VEGF staining was negative. (B) 4 months old LDL receptor deficient mice (C57BI/6J-LDL-r(-/-)) after 2 months of high fat diet. VEGF staining is positive (intense red) in retinal pigment epithelium (RPE), outer plexiform layer (OPL), inner photoreceptor segments (IPS).

VEGF expression was documented in all six LDL receptor deficient mice following HF diet.

\section{DISCUSSION}

The LDL receptor knockout model serves as an established model for atherosclerotic pathomechanisms because of the opportunity to induce elevated pTC levels.

In our study pTC was significantly increased by LDL receptor deficiency as well as HF diet. LDL receptor deficient mice after HF diet showed the highest average pTC, which was 6.0 times more than in $\mathrm{C} 57 \mathrm{Bl} / 6 \mathrm{~J}$ control mice consuming standard chow diet.

Furthermore, we demonstrated a structural degeneration of BrM with thickening and accumulation of membrane and non-membrane bound translucent particles in LDL receptor deficient mice correlating with significantly elevated pTC levels. The layer arrangement was affected as well. These degenerations were most prominent in LDL receptor deficient mice after HF diet. Hypercholesterolaemia as an atherosclerotic risk factor seems to predispose to these structural alterations in BrM.

The observed changes in murine BrM resemble those seen in human eyes of aged donors and donors with ARM. ${ }^{11-13}$ Electron lucent droplets in adult human eyes are also scattered throughout BrM and form in elderly eyes a discrete layer external to the RPE basal lamina. ${ }^{4}$ Membrane bounded vesicles are the principal component of basal linear deposits and large drusen. The presence of such lipid deposits is associated with early and late ARM. ${ }^{4}$ There is evidence in humans that the observed translucent vesicular changes are not vesicles, but solid neutral lipid rich particles extracted by regular tissue processing for TEM. ${ }^{3}{ }^{14}$ In histological studies such particles bind oil red $\mathrm{O}$ and contain esterified cholesterol and triglyceride. ${ }^{514}$ The electron lucent droplets in basal linear deposits resemble extracellular material found in atheromatous plaques. ${ }^{15}$ In general, human BrM seems to undergo ageing processes similar to the arterial intima layer and other connective tissues for which plasma lipoproteins are the known source of extracellular cholesterol. ${ }^{3}{ }^{15}$

$\mathrm{BrM}$ is a connective tissue composed of five layers. It is responsible for many transport and support functions between RPE and choriocapillaris and is therefore essential for the health of the entire retina. Accumulation of lipids in BrM is believed to alter its diffusion characteristics and potentially compromises the metabolic exchange between choroid and retina affecting photoreceptor function. ${ }^{1}$

Expression of VEGF was detectable exclusively in LDL receptor deficient mice. Most intensive VEGF staining was found in LDL receptor deficient mice following $\mathrm{HF}$ diet corresponding to the increased amount of vesicles in this group. VEGF was detected in RPE, photoreceptor inner segments, and the outer plexiform layer.

VEGF is an angiogenic growth factor which is known to be a major stimulator for ocular neovascularisation. ${ }^{12}$ The two smaller VEGF isoforms 165 and 121 are secreted proteins and act as diffusible agents while the two larger isoforms 189 and VEGF 206 remain cell associated. Predominantly, the RPE is known to secret VEGF supporting paracrine and autocrine functions. ${ }^{1}$ A major pathomechanism for increased VEGF expression in the pathogenesis of CNV is not clearly identified yet. ${ }^{2}$ Unlike retinal neovascularisation, it is not apparent that general hypoxia is an important factor for upregulation of VEGF for CNV development. ${ }^{216-19}$ Cumulative oxidative stress caused by reactive oxygen intermediates occurring as byproducts of cellular metabolism and photochemical reactions as well as advanced glycation end products may have a role. ${ }^{16}{ }^{17} 20$ Compromised diffusion via lipid rich, hydrophobic BrM may cause increased oxidative stress by accumulating metabolic end products. ${ }^{120}$ It is beyond the scope of this pilot study to determine the cause of more intensive VEGF expression. However, an increased VEGF expression in relation to advanced alterations of BrM caused by elevated plasma cholesterol levels was clearly observed. In our model no spontaneous CNV was observed, but no substantial breaks in BrM either, which is a supposed prerequisite. Increased thickness and hydrophobicity of BrM by lipids may prevent VEGF from reaching the choriocapillaris and stimulating CNV. ${ }^{1}$ A break in BrM by, for example, advanced degeneration definitively would overcome this barrier and CNV may develop. These observations appear to bridge the gap between hypercholesterolaemia and BrM changes as well present in parts of a possible progression mechanism of early ARM to neovascular stages.

\section{CONCLUSION}

Atherosclerosis and ARM appear to be complex diseases with many environmental and genetic factors. In part, they share common risk factors and common animal models can be used to learn more about these age related degenerative diseases.

\section{Authors' affiliations}

M Rudolf, Department of Ophthalmology, University of SchleswigHolstein, Campus Kiel, Germany

B Winkler, Department of Ophthalmology, University of SchleswigHolstein, Campus Luebeck, Germany

Z Aherrahou, L C Doehring, P Kaczmarek, Atherosclerotic Study Group, Department of Medicine, University of Luebeck, Germany

U Schmidt-Erfurth, Department of Ophthalmology, University of Vienna, Austria

Competing interests: none declared

Correspondence to: Dr med Martin Rudolf, University of SchleswigHolstein, Campus Kiel, Department of Ophthalmology, HegewischStrasse 2, 24105 Kiel, Germany; mirudolf@aol.com

Accepted for publication 1 July 2005 


\section{REFERENCES}

1 Ambati J, Balamurali K, Ambati K, et al. Age-related macular degeneration: etiology, pathogenesis, and therapeutic stragegies. Surv Ophthalmol 2003;48:257-92

2 Campochiaro RA. Retinal and choroidal neovascularization. J Cell Physiol 2000;184:301-10.

3 Curcio AC, Millican CL, Bailey T, et al. Accumulation of cholesterol with age in human Bruch's membrane. Invest Ophthalmol Vis Sci 2001:42:265-73.

4 Curcio AC, Millican CL. Basal linear deposits and large drusen are specific for early age-related maculopathy. Arch Ophthalmol 1999;117:329-39.

5 Pauleikoff D, Harper CA, Marshall J, et al. Aging changes in Bruch's membrane: a histochemical and morphological study. Ophthalmol 1990;97:171-8

6 Vingerling JR, Dielemans I, Bots ML, et al. Age-related macular degeneration is associated with atherosclerosis. The Rotterdam Study. Am J Epidemiol 1995; 142:404-9.

7 Snow K, Seddon J. Do age-related macular degeneration and cardiovascular disease share common antecedents? Opthalmic Epidemiol 1999;6:125-43.

8 The Eye Disease Case Control Study Group. Risk factors for neovascular agerelated macular degeneration. Arch Ophthalmol 1992;110:1701-1708.

9 Rudolf $M$, Ivandic B, Winkler J, et al. Akkumulation von Lipidpartikeln in der Bruchmembran von LDL-Rezeptor-defizienten Mäusen als Modell für die Altersbezogene Makuladegeneration. Ophthalmol 2004;101:715-19.

10 Kwak N, Okamoto N, Wood JM, et al. VEGF is major stimulator in model of choroidal neovaskularisation. Invest Ophthalmol Vis Sci 2000;41:3158-64.
11 Sheraidah GS, Steinmetz R, Maguire J, et al. Correlation between lipids extracted from Bruch's membrane and age. Ophthalmology 1993; 100:47-51

12 Green WR, Enger C. Age-related macular degeneration histopathologic studies. Ophthalmology 1993;100:1519-35.

13 Dithmar S, Curcio AC, Le HA, et al. Ultrastructural changes in Bruch's membrane of apolipoprotein E-deficient mice. Invest Ophthalmol Vis Sci 2000;41:2035-42.

14 Holz FG, Sheraidah G, Pauleikoff D, et al. Analysis of lipid deposits extracted form human macular and peripheral Bruch's membrane. Arch Ophthalmol 1994;112:402-6.

15 Bocan TM, Schifani TA, Gyuton JR. Ultrastructure of human aortic fibrolipid lesion: formation of the atherosclerotic lipid-rich core. Am J Pathol $1986 ; 123: 413-24$

16 Kuroki $M$, Voest EE, Amano $S$, et al. Reactive oxygen intermediates increase vascular endothelial growth factor expression in vitro and in vivo. $J$ Clin Invest 1996;98:1667-75

17 Lu M, Kuroki M, Amano S, et al. Advanced glycation end products increase retinal vascular endothelial growth factor expression. J Clin Invest 1998;101:1219-24.

18 Wada M, Ogata N, Otsuji T, et al. Expression of VEGF and its receptor (KDR/ flk-1) mRNA in experimental choroidal neovascularization. Curr Eye Res 1999:18:203-13.

19 Stone J, Itin A, Alon T, et al. Development of retinal vasculature is mediated by hypoxia-induced VEGF expression by neuroglia. J Neurosci 1995; 15:4738-47.

20 Beatty S, Koh H, Phil M, et al. The role of oxidative stress in the pathogenesis of age-related macular degeneration. Surv Ophthalmol 2000;45: 115-34. 